\title{
Evasión tributaria, una revisión ${ }^{1}$
}

\author{
Ruth Alejandra Patiño Jacinto ${ }^{2}$ \\ Sandy Tatiana Mendoza Gómez $z^{3}$ \\ Diego Alexander Quintanilla Ortiz ${ }^{4}$ \\ José Díaz Montenegro ${ }^{5}$
}

Recibido: 28 de mayo de 2019

Aprobado: 27 de junio de 2019

1 Artículo de investigación desarrollado en el año 2018.

Patiño Jacinto, R. A., Mendoza Gómez, S. T., Quintanilla Ortiz, D. A., \& Díaz Montenegro, J. (2019). Evasión tributaria, una revisión. Revista Activos, 17(1), 167194. DOI: https://doi.org/10.15332/25005278.5399

2 Contadora pública egresada de la Universidad Nacional de Colombia, magíster en Ciencias Económicas de la Universidad Santo Tomás, estudiante de Doctorado en Educación en la Universidad Santo Tomás. Líder del grupo de investigación Contaduría: Información, Control e Impacto Social. Correo electrónico: alejandrapatino@usantotomas.edu.co

3 Contadora pública egresada de la Universidad Santo Tomás, maestrante en Administración de Empresas con Especialidad en Gerencia de Proyectos de la Universidad Arturo Prat. Docente e investigadora del Programa de Contaduría Pública de la Corporación Universitaria Minuto de Dios - Uniminuto. Integrante del grupo de investigación Educación, Sujeto y Cultura. Correo electrónico: sandy. mendoza.g@uniminuto.edu

4 Contador público y magíster en Administración, egresado de la Universidad Nacional de Colombia, docente de la Facultad de Contaduría Pública de la Universidad Santo Tomás, Bogotá. Líneas de desarrollo: Contabilidad de Gestión, Educación Contable, Hacienda Pública. Correo electrónico: diegoquintanilla@ usantotomas.edu.co

5 Contador público y economista, egresado de la Universidad de Especialidades Espíritu Santo y de la Universidad de Guayaquil, Ecuador. Estudiante de doctorado en Sostenibilidad en la Universidad Politécnica de Catalunya (UPC), España. Integrante del grupo de investigación: Business Innovation and Technology Management (BITM) de la Universidad Estatal de Milagro, Ecuador, e investigador en formación del Centre de Recerca en Economia i Desenvolupament Agroalimentari, Creda-UPC. 


\title{
Clasificación JEL: H26
}

\section{Resumen}

El presente artículo tiene como temática la evasión tributaria, la cual es un problema mundial, de ahí su importancia y relevancia en investigación. En el presente artículo se realiza una revisión general sobre el comportamiento social, de valores y tributario de los contribuyentes y busca dar algunas explicaciones de los fenómenos de la corrupción y la evasión, casos específicos los paraísos fiscales y centros financieros extraterritoriales, evasión medida desde la información financiera de las entidades financieras y medición de la evasión con respecto al sistema fiscal.

Palabras clave: evasión tributaria, impuestos, corrupción, política fiscal.

\section{Tax evasion, a review}

\begin{abstract}
This article has as its theme tax evasion, which is a worldwide problem, hence its importance and relevance in research. In this article a general review is carried out on the social behavior, values, taxpayers' taxpayers and seeks to give some explanations of the phenomena of corruption and evasion, specific cases, tax havens and extraterritorial financial centers, evasion measured from the financial information of financial institutions and measurement of evasion with respect to the tax system.
\end{abstract}

Keywords: Tax evasion, Taxes, Corruption, Fiscal policy. 


\section{Introducción}

En sociedades como las latinoamericanas en las cuales se han evidenciado limitaciones en las políticas públicas relacionadas con la distribución del ingreso y con la destinación del gasto público, también se ha cuestionado la estructura tributaria de estos países en los cuales se han adoptado estrategias directas de distribución por medio de gastos y en la eficiencia en el recaudo, para mitigar estos problemas.

Los hechos anteriores forman parte de la política tributaria, la cual además tiene impacto desde la selección de impuestos directos como la renta, tributo en el cual paga el que tiene la capacidad (progresivos), o de los indirectos como el caso del IVA, los cuales son regresivos. De esta forma, para impactar la redistribución, se generan recursos para financiar el Estado (Jiménez, Gómez y Podestá, 2010).

Dada la escasez de recursos para cubrir las necesidades de presupuesto de recursos públicos se genera una preocupación por la evasión fiscal, es decir, entendida como:

Un hecho, el cual se basa en el incumplimiento de obligaciones tributarias, el no pago, generada por diversos fenómenos de índole social, político y económico, tales como la falta de credibilidad del Estado, la naturaleza del individuo de pagar lo menos posible, la excesiva carga tributaria, los ciclos económicos, entre otros. (Parra y Patiño, 2010, p. 181)

La evasión tributaria es relevante como tema de estudio dado que este problema distorsiona el funcionamiento del sistema tributario. El pago de impuestos está ligado a la redistribución de la riqueza, de tal forma que, si se diseña de manera adecuada, a través de impuestos y transferencias, es un instrumento poderoso para contribuir a impulsar una mayor igualdad y un crecimiento económico y social (OECD, 2015).

La evasión como un fenómeno social se da por múltiples causas, entre ellas la desconfianza y la inconformidad de los contribuyentes con el 
sistema tributario, por ello distintos autores proponen estrategias con el fin de mejorar el sistema tributario. En el caso de la Subdirección General de Asuntos Económicos y Financieros de la UE (2013), esta menciona la necesidad de devolver a los ciudadanos la confianza y paralelamente realizar cruce de información entre países como un sistema de control tributario.

Como se evidencia, la evasión es un tema de impacto económico, por ello la preocupación por lograr una cuantificación del fenómeno, lo que en últimas sirve de referente para las decisiones de política fiscal. Tomando la clasificación que proponen Shome y Tanzi (1993), los métodos para medir la evasión se dividen en directos e indirectos. Entre los métodos directos se pueden identificar el uso de las variables macroeconómicas como el ingreso; los métodos indirectos se miden por medio de variables microeconómicas con muestras de contribuyentes, por ejemplo: método muestral de auditorías y el método de las encuestas directas de opinión a los contribuyentes (Cosulich, 1993). Por su parte, Piolatto (2014) expresa que la evasión de impuestos es una preocupación de la mayoría de los países que por su naturaleza es difícil de estimar, y Allingham y Sandmo (1972) consideran que la evasión de los impuestos es una decisión en riesgo.

Para Cerqueti y Coppier (2011), citados por Sidani, Ghanem y Rawwas (2014), la evasión de impuestos es un problema de las sociedades desarrolladas y en desarrollo que tiene implicaciones sobre el crecimiento económico, reduciendo la posibilidad que tienen los gobiernos para proveer los servicios públicos (p. 183), dado que disminuyen los recursos públicos.

Nickerson et al. (2009), citados por Sidani, Ghanem y Rawwas (2014), indican que existen diversas perspectivas de evadir impuestos: algunas personas lo hacen por egoísmo, otras lo hacen cuando observan que el sistema tributario no funciona correctamente y detectan la corrupción, y otras cuando sienten que el sistema no opera con equidad en las diversas clases sociales; es decir, que hay una estrecha relación con la legitimidad.

La evasión de los impuestos puede ser persuadida a través de la detección y las sanciones (Franzoni, 1999), los consumidores racionales 
deciden si evadir o no el pago de impuestos sobre la base de comparación de costo-beneficio; mientras las autoridades fiscales luchan, los instrumentos disponibles son limitados, tal es el caso de la reducción de los beneficios de la evasión (reducción de tasas de impuestos) y el aumento de los costos (multas elevadas y sistemas de auditorías sofisticadas) (Piolatto, 2014). Todo esto forma parte de la decisión del contribuyente.

Lo anterior se confirma con lo que señalan (Parra y Patiño, 2010) al decir que el Estado cuenta con diferentes tipos de política, tales como la política fiscal, que es una de las herramientas más importantes para cumplir con objetivos macroeconómicos y microeconómicos. La evasión tributaria distorsiona estas decisiones que implican problemas de política fiscal, reflejados en aumentos de impuestos y pagos injustificados para ciudadanos y empresarios. Esto demuestra que corresponde a un asunto relevante que merece una discusión adecuada.

El presente documento incluye la metodología, posteriormente muestra los resultados de la revisión de la evasión tributaria, partiendo de unas generalidades; luego, una breve discusión de la relación del tema con los valores y la corrupción, después se analizan casos específicos de evasión tributaria, los paraísos fiscales extraterritoriales, la evasión medida desde la información de las entidades financieras y el problema de la medición; por último, se hacen unas reflexiones a manera de conclusión.

\section{Metodología}

Para la construcción de este artículo se parte de un enfoque cualitativo, ya que corresponde a "cualquier tipo de investigación que produce hallazgos a los que no se llega por medio de procedimientos estadísticos u otros medios de cuantificación" (Strauss y Corbin, 2002, p. 19), es decir, se trabaja a partir de una revisión de características; en este caso se pretende conocer y caracterizar las publicaciones en temas de evasión, lo cual conlleva establecer los avances del tema en diferentes entornos. 
Por otra parte, la investigación se basa en "métodos de recolección de datos no estandarizados ni completamente predeterminados" (Hernández, Collado y Baptista, 2010, p. 9) y en "la investigación que produce y analiza los datos descriptivos, como las palabras escritas o dichas" (Deslauriers, 2004, p. 6). En este sentido, se plantea la recolección y sistematización de documentos teóricos con respecto a la evasión, lo cual tiene por objetivo establecer un inventario de los avances en el tema, para luego identificar categorías, las cuales se constituyeron luego de la indagación en los documentos. Es una investigación documental textual, la cual se desarrolla a partir de artículos académicos en revistas indexadas y abarca las siguientes fases:

1. Se realizó una búsqueda en la base de datos de SCImago en la cual se encuentran las revistas internacionales indexadas, se filtró por áreas y se seleccionó la categoría de economía en la que aparecen 880 revistas, de estas se excluyeron las que corresponden a temas muy específicos como contabilidad, finanzas, economía laboral y mercadeo, entre otros, por lo cual quedan 412 revistas para realizar búsqueda bibliográfica. En asuntos relacionados con evasión tributaria, se trabajó con revistas entre 2015 y 2019.

2. Con el fin de lograr una aproximación al contexto latinoamericano se realiza la búsqueda de revistas de economía en Latindex, donde luego de depurar las que corresponden a temas específicos como en la fase anterior, se encuentran 179 revistas para hacer la búsqueda con el tópico. Nuevamente se indaga en las publicaciones de 2015 a 2019.

3. De la revisión previa se logró identificar 67 artículos de temáticas relacionadas con evasión tributaria agrupados en cinco grandes categorías, las cuales inicialmente se verificaron en el resumen y las palabras clave; dichas categorías se analizan y se presentan en los resultados.

\section{Resultados}

\subsection{Una revisión general}

Se han realizado múltiples aportes con respecto a la evasión tributaria, estudios de causas y de comportamientos con respecto al sistema fiscal, 
entre otros. Algunos de los principales aportes en la revisión bibliográfica realizada se presentan a continuación:

De acuerdo con Bernasconi, Levaggi y Menoncin (2016), el comportamiento humano con respecto a la evasión es variable con el tiempo; por ejemplo, a mayor edad es menor la probabilidad de evadir, por lo tanto, es más eficiente para las autoridades tributarias controlar y no multar. Respecto a las tasas, cuando estas aumentan también lo hace el nivel de evasión. En cuanto al control de la evasión, Kuchumova (2017) mediante un trabajo empírico desarrolla la idea de que la auditoría tributaria es el medio de control fiscal más efectivo.

En relación con la evasión tributaria, McGee, Devos y Benk (2016) mencionan que en un estudio comparativo entre Turquía y Australia hallan que la evasión tributaria se justifica desde la perspectiva ética, es decir, que la actitud cultural frente a un sistema tributario injusto e inequitativo, y en el cual no se gaste o invierta en aspectos primordiales, se considera válido evadir impuestos. Ferrer y Gerxhani (2016) plantean que la evasión se reduce cuando el bienestar y la percepción de las instituciones es positiva.

López y Sanz (2016) explican las características individuales, sociales y político-institucionales que inciden en el cumplimiento de las obligaciones fiscales, los autores mencionan que dentro de las características que tienen una relación positiva con el cumplimiento de tipo personal están: mayor edad, mujeres, casados, alto nivel educativo, sentirse feliz, tener satisfacción financiera, orgullo nacional, altruismo y practicante de alguna religión, entre otros; por otra parte, consideran que los factores que coinciden con características de evasor son: propensión al riesgo, desarrollar actividades independientes, alto nivel de renta, etc. En lo social identifican que en un sistema con evasión alta tiende a incrementarse, así como la inequidad fiscal también tiene un impacto al aumentar la evasión. En lo relacionado con el gasto público, de acuerdo con la percepción de si es indebido o no, incrementa o disminuye el no pago de impuestos; en las variables políticas e institucionales identifican como variables inversas con la evasión tributaria: la confianza sobre el Gobierno e instituciones como la policía, además de 
la democracia, descentralización y gestión pública eficiente, entre otros, mientras que se observa como factores negativos los costos para cumplir las obligaciones y amnistías fiscales.

Definitivamente el papel de los agentes de gobierno en la evasión en muy relevante: en estudios previos se ha encontrado entre las causales algunos asociados con las decisiones de política; es así como los agentes políticos tienen relación con el cumplimiento de la obligatoriedad fiscal por medio de incentivos, el uso de tecnología en el control y cruce de información, además de la eficiencia, proporcionando bienes públicos y la percepción de esto en lo social (Casaburi y Toriano, 2015).

Para Banerjee y Vaidya (2018) la amenaza de acoso por parte del funcionario tributario hacia el contribuyente puede aumentar de manera contraintuitiva el impacto de las reformas de anticorrupción en evasión de impuestos, en especial en la penalización de la anticorrupción que no solo elimina la corrupción sino también induce una reducción discontinua en la evasión fiscal a un nivel incluso más bajo que el que podría lograrse cuando los oficiales actúan legalmente.

Lo anterior se confirma con lo señalado por Dularif, Nurkholis y Saraswati (2019), quienes afirman que la auditoría y penalización crean un impacto positivo en el cumplimiento tributario, mientras que el aumento de la tasa impositiva tiende a inducir evasión fiscal.

Abdixhiku, Pugh y Hashi (2018) corroboran que los factores institucionales y de la tasa impositiva: la mayor corrupción, las reformas más lentas y las tasas impositivas más altas, combinados reducen sustancialmente la cantidad de impuestos pagados por las empresas.

Respecto al control fiscal llevado a cabo por las autoridades de gobierno afirman que en el caso particular de Rumania (Ionel, 2016), acciones como el cruce de información han disminuido significativamente la evasión, con lo que luego reducen los recursos públicos destinados en controlar la evasión. 
Sin embargo, para Porter y Ronit (2018) los estados pueden actuar espontáneamente y no construir la capacidad necesaria para impulsar el papel de los gobiernos en la lucha contra la evasión fiscal e incluso obstaculizar las iniciativas intergubernamentales.

\subsection{Comportamiento social, valores, corrupción y evasión tributaria}

Para abordar el presente tema es importante citar la investigación realizada por McGee, Yoon y Li (2015) quienes identifican que diversos argumentos han sido formulados a lo largo de los siglos para justificar la evasión de impuestos; una demostración ha sido el caso en que la gente percibe que el sistema impositivo es injusto, el Gobierno es corrupto, las tasas de impuestos son demasiado altas, hay una incapacidad para pagar, gran parte de los fondos recaudados se pierden o terminan en los bolsillos de los políticos corruptos, o de sus amigos o familiares; los fondos se destinaron a proyectos que el contribuyente moralmente desaprueba, o cuando los contribuyentes perciben que no están obteniendo una retribución en sus pagos de impuestos. Lo anterior fomenta una percepción de que la evasión fiscal es aceptable, si los demás hacen o si la probabilidad de ser descubierto es baja.

Para Saxunova y Szarkova(2018) existe falta de voluntad para pagar impuestos o diversas tarifas. La evasión fiscal representa un grave problema de cada economía y tiene efecto negativo en el presupuesto estatal; de ahí que la evasión fiscal es una amenaza para la sociedad, los estados y las organizaciones internacionales, que han estado haciendo esfuerzos para combatir los fenómenos negativos asociados con la tributación, la evasión o el fraude fiscal.

Es así como las causas de la evasión de impuestos radican en niveles internos o individuales y contextuales, sociales o institucionales (LagoPeñas y Lago-Peñas, 2010) citados por (Alarcón, Buendía y Sánchez, 2017), es decir, desde la conducta de cumplimiento individual en función de los valores (Porcano, 1988; Erard y Feinstein, 2004; Molero y Pujol, 2005) 
citados por Alarcón et al. (2017), y desde aspectos institucionales, sociales y las diferencias económicas entre países y regiones (Torgler, 2003, citado por Alarcón et al., 2017). Alm (2013), citado por Alarcón et al. (2017), señala un tercer nivel que mezcla a un grupo de motivaciones, como el altruismo o la equidad. Asimismo, Torgler (2005) indica que cuando los contribuyentes participan directamente en las decisiones políticas, el contrato fiscal es más fuerte y es basado en la confianza, lo que hace que la moral fiscal aumente. Kirchler, Hoelzl y Wahl (2008) y Kirchler y Wahl (2010) dicen que el poder y la confianza en las autoridades fiscales son aspectos relevantes para la comprensión de desapariciones forzadas y de cumplimiento voluntario. Alarcón et al. (2017) llegan a la conclusión conclusión de que factores sociodemográficos y socioeconómicos afectan la moral de los impuestos, tales como los ingresos, el gasto público, la inflación, el desempleo, la edad, el sexo, la situación económica, la religión y la confianza en la justicia y los sistemas parlamentarios.

Para Sunstein (1996), citado por Gokalp, Leeb y Pengb (2017), la legalidad o ilegalidad de una acción recae sobre los encargados de formular políticas que afectan la aceptación social. Ante esto, Nwabuzor (2005) expresa que eludir las regulaciones del Gobierno puede ser engorroso, debido a la tediosa burocracia y corrupción.

Lo anterior se confirma desde la perspectiva en las actividades de negocio. Zhang (2011) explica que los informes fraudulentos en documentos de comercio internacional están estrechamente vinculados a las firmas, con el fin de ir tras los incentivos fiscales preferenciales otorgados a los inversionistas extranjeros. Desde una visión basada en la institución, el costo de reproducción de las reglas del juego se hace mayor cuando la carga tributaria es mayor, de ahí que el aumento de la carga fiscal hará que las firmas intenten eludir pasivos y oculten parte de sus ingresos al Gobierno (Gokalp et al., 2017).

Beck, Lin y Ma (2014) mostraron que las firmas en países con sistemas eficaces de intercambio de información crediticia y con mayor penetración de sucursales bancarias son menos propensas a evadir el pago de impuestos, 
encontraron el efecto más fuerte para las pequeñas firmas, y se llegó a la conclusión de que un sistema financiero que facilita el acceso al crédito aumenta el costo de oportunidad de la evasión de impuestos (Gokalp et al., 2017).

Asimismo, McGee et al. (2015) plantean que es más fácil evadir el pago de impuestos en algunos países y en algunas profesiones, y es casi imposible para aquellos donde el Gobierno tiene una política de retención de impuestos de los cheques de pago; donde no todo el mundo tiene un sueldo y los independientes suelen tener la opción de evadir algunas o todas sus obligaciones tributarias al igual que los inversionistas; aspectos que tienen que ver con la oportunidad y la obligación moral de pagar.

De acuerdo con lo indicado por Zimmerman (1983), citado por Watson (2015), las empresas exitosas están sujetas a altos costos políticos, conducidas a mayores pagos de impuestos (p. 5). Para Argentiero, Chiarini y Marzano (2015, p. 2) surge el interrogante de “¿Por qué la evasión fiscal puede ser considerada como un factor determinante de los delitos económicos?". Además, se afirma que las actividades delictivas podrían ser indirectamente conformadas por evasión fiscal, pues una de las razones es la redistribución de ingresos que la evasión fiscal genera hacia cualquier ingreso que no es claramente atribuible; así mismo la carga tributaria a las pequeñas empresas, artesanos, comerciantes, mercaderes y profesionales ha producido fuertes desigualdades que pueden haber repercutido en la actividad criminal. De igual forma la fuerte redistribución del ingreso hacia empleados y pequeñas empresas origina un inevitable incremento de la desigualdad produciendo delitos contra la propiedad. Adicionalmente los autores señalan que el efecto riqueza impulsa en los territorios a cometer más delitos y el crimen impulsa al crecimiento de la informalidad, ya que el trabajo legal es poco lucrativo, lo que promueve las actividades clandestinas y la cultura de la ilegalidad.

Argentiero et al. (2015) reiteran que los factores antes mencionados impactan positivamente sobre el delito de evasión de impuestos y añaden que cuando la evasión fiscal se coloca en relación con determinados delitos 
económicos como la usura y los fraudes, causa un aumento en los ingresos no declarados a las autoridades fiscales, que pueden afectar negativamente a la delincuencia ya que estos fenómenos contribuyen también a financiar actividades legales de los hogares y las pequeñas empresas. En este sentido mencionan que una de las razones por las que la evasión de impuestos puede afectar a los delitos contra la propiedad, es que cuando las víctimas de estos crímenes son evasoras de impuestos, tienen menos incentivos para reportar el crimen sufrido, lo cual genera una baja probabilidad de capturas y sentencias.

Ante estos eventos, Argentiero et al. (2015) concluyen que la usura y los fraudes a la evasión fiscal representan una fuente alternativa de financiación con respecto al trabajo ilegal o financiación criminal, mientras que los delitos contra la propiedad aumentan la evasión fiscal y ocasionan un crecimiento de la delincuencia. Así pues, cuanto mayor sea la carga tributaria, más la persona elige la evasión de impuestos en lugar de usura y fraudes. Teniendo en cuenta el tema ya abordado es importante destacar lo citado por Maji (2017, p. 3), en cuanto a que la injusticia solo debilita el sistema tributario (Gilligan y Richardson, 2005) y motiva a los contribuyentes a no cumplir con el sistema tributario (Robert y Hite, 1994).

De igual forma, Fochmann y Wolf (2019) señalan que los sujetos están sistemáticamente más dispuestos a evadir impuestos que a subinformar los ingresos positivos (por ejemplo, ingresos de negocios o no comerciales) e ingresos negativos (como deducciones, créditos o pérdidas). En segundo lugar, la compensación de ingresos positivos y negativos reduce el comportamiento asimétrico de evasión fiscal. Tercero, la interacción de los ingresos tiene un papel importante en las decisiones de evasión fiscal, lo cual enfatiza que el diseño de las declaraciones de impuestos puede tener una grave influencia en el comportamiento de evasión fiscal.

Un requisito previo para el diseño de políticas efectivas es poder comprender, en términos cuantitativos, el comportamiento de los evasores de impuestos (Goumagias, Hristu-Varsakelis y Assael, 2018). 
Sin embargo, para Zídková, Tepperová y Helman (2018) comprender el estado de la moral tributaria es útil cuando se implementa un sistema eficiente y un sistema tributario equitativo, de ahí que no se puede abordar la inclinación a evadir impuestos solo por análisis cuantitativo desde motivos subjetivos y suposiciones de individuos ya que estos factores personales interactúan con las normativas legales y los valores sociales de forma intrincada.

Para concluir el presente tema y teniendo en cuenta la literatura abordada, se puede inferir que cada país tiene sus propias políticas fiscales, que permiten al ser humano tener conductas variantes frente a la evasión de los impuestos. Ante un sistema tributario robusto y un Estado transparente, el contribuyente tiende a tener comportamientos de legalidad y compromiso frente al pago de los impuestos, lo que permite disminuir prácticas de corrupción y actividades criminales. Cuando los Estados adoptan prácticas sanas en la distribución de los recursos e implementan incentivos tributarios a los ciudadanos, los países tienden a ser prósperos, por tanto los niveles de desempleo son bajos y el nivel de vida es óptimo.

\subsection{Casos específicos de evasión tributaria}

Nygard, Slemrod y Thoresen (2016) señalan que la evasión puede beneficiar tanto a compradores como a vendedores. El comprador accede a un bien o servicio a un menor precio y el vendedor reduce su carga tributaria. Las implicaciones distributivas de este tipo de evasión dependen de la frecuencia y cantidad de transacciones con estas características. Estos investigadores afirman que en Noruega la desigualdad entre los ingresos reportados y los ingresos obtenidos tiene una mayor dispersión a las estimaciones oficiales de ese país.

Bobbio (2016) realizó una investigación en la cual establece que uno de los impactos de la evasión en Italia es el lento crecimiento de la economía en este país. Lo anterior lo atribuye a que las pequeñas y medianas empresas tienen una baja inversión en investigación y desarrollo porque 
este tipo de inversiones conllevan no solamente los costos asociados a este tipo de actividades, sino que hace que estas salgan de la sombra. Este tipo de aseveraciones surgen al señalar que la probabilidad de auditar los impuestos de una compañía aumenta a medida que van creciendo. Dicha investigación manifiesta que al analizar el nivel empresarial entre el periodo comprendido entre 1995 y 2006, que este estímulo a la evasión ha ocasionado que la economía de este país deje de crecer en un $0.9 \%$ a un $1.1 \%$; de igual forma la participación de estas compañías hubiese sido un $25 \%$ más alto en la economía.

Goerke, Bonn y München (2016) realizan una investigación tomando de referencia un oligopolio de Cournot ${ }^{6}$, en la cual señalan que la evasión puede agravar o mitigar la entrada excesiva de empresas a un mercado; también se evidencia que la evasión genera un mal en el mercado dado que disminuye la utilidad esperada por parte de los oferentes. Lo anterior se establece al señalar que la evasión de impuestos puede fomentar la entrada de participantes a un mercado, porque al evadir el impuesto aumenta la ganancia por parte del evasor; sin embargo, el autor menciona que, al hacer un análisis más detenido, el evadir impuestos conlleva recursos, lo cual se verá manifestado en las utilidades de las empresas del mercado. Por último, argumenta que un impuesto a la ganancia no distorsiona la elección del nivel de producción de una compañía.

Henry, Massel y Towery (2016) por su parte, al realizar un análisis en el cual comparan las empresas nacionales y las internacionales, expresan que los niveles de divulgación y la posibilidad de elusión o evasión difieren entre estos dos tipos de entidades, aunque sostienen que la probabilidad de evasión en una empresa multinacional, evidencia que la evasión fiscal parece ser más alta en los últimos años analizados, cuando estos años concuerdan con un mayor grado de exigencia en los reportes de las transacciones

6 Un oligopolio de Cournot es un modelo microeconómico, el cual describe una estructura de industria en la cual las empresas de un mercado compiten en el nivel de producción de unos bienes homogéneos. Este modelo de decisión toma como premisa la maximización de utilidades y la asimetría de información en relación con el nivel de producción de los participantes del mercado. 
realizadas por las compañías, de tal forma que esta investigación evidencia la necesidad de revisar los sistemas de divulgación de información existentes.

Hoopes, Thornock y Williams (2016) identifican que los minoristas electrónicos no recaudan impuestos sobre las ventas de la mayoría de las transacciones realizadas, lo cual genera una ventaja competitiva sobre los minoristas tradicionales; esta situación puede cambiar ante una legislación más fuerte sobre este impuesto y las ventas electrónicas.

Lo anterior se confirma por parte de Immordino y Russo (2018), quienes indican que fomentar el uso de pagos sin efectivo podría ayudar a combatir la evasión fiscal y como estrategia implica subsidiar el uso de tarjetas de crédito y débito, realizadas, por ejemplo, a través de reembolsos basados en el volumen de transacciones; lo anterior dado que el uso de tarjetas de pago para retirar dinero en cajeros automáticos en realidad está asociado con más evasión de impuestos. Al igual que otra posibilidad que plantean sería subvencionar inversiones en infraestructura de banda ancha, promoviendo así el comercio electrónico y, en consecuencia, el uso de pagos electrónicos.

\subsection{Los paraísos fiscales y centros financieros extraterritoriales}

Mityakov (2015) realizó un análisis a partir de los informes obligatorios de los bancos rusos al organismo central para establecer el impacto de la banca extraterritorial. Este análisis evidencia una relación positiva entre la evasión y las transacciones hechas mediante banca extraterritorial; de igual forma, se evidencia que estas actividades también tienen una relación positiva con otras actividades ilícitas como son el lavado de dinero y el fraude contable.

Los autores atribuyen que este trabajo tiene tres resultados significativos: en primera instancia establecen medidas de participación entre los bancos extraterritoriales y las firmas financieras y no financieras; en segunda instancia evidencian la evasión fiscal de los bancos y las empresas rusas; y en tercer lugar buscan evidenciar la supervivencia a largo plazo de 
los bancos que realizan actividades con jurisdicciones más transparentes. Lo anterior lo logran al analizar los informes que presentan las entidades financieras al banco central, tomando como período de análisis desde el año 2000 hasta el 2003. Este tipo de informes les permitió analizar el volumen de transacciones y los saldos de las cuentas de los bancos rusos en todos los países extranjeros. Para las entidades no financieras los autores analizaron un conjunto de datos de transferencias realizadas a través de bancos comerciales rusos entre 1998 y 2004.

Los resultados de esa investigación tienen un alto nivel de detalle, a tal punto que identifican una sólida relación entre las actividades bancarias extraterritoriales y la evasión fiscal por altos directivos de los bancos, lo cual lleva a tener una relación negativa entre los bienes declarados y la actividad bancaria en entidades extraterritoriales. Estas relaciones también se identifican en el sector no financiero que realiza transacciones con bancos extraterritoriales.

Pellegrini, Sanelli y Tosti (2016) analizan la incidencia de los paraísos fiscales y de los Centros Financieros Extraterritoriales ${ }^{7}$ en la evasión internacional. Para lo anterior, en primera instancia realizan una estimación del capital no reportado tomando como referencia los ingresos fronterizos de entidades no bancarias. Con base en esta estimación identifican que, aproximadamente, entre 2001 y 2013 la evasión del impuesto sobre la renta en capital se encuentra entre \$19 y \$38 000 millones; lo anterior entendiendo que los activos no declarados son ingresos no declarados sobre la renta personal, de tal forma que se estima que el impuesto a la renta evadida puede estar entre $\$ 2.0$ y $\$ 2.6$ billones. Los dos ejercicios de estimación se hacen en el ámbito global, distinguiéndose entre países de la Ocde y los no pertenecientes a ella, y con respecto al caso italiano.

7 Los centros financieros extraterritoriales son entidades que se encuentran en jurisdicciones o países en donde el sector financiero tiene una participación significativa de la economía de ese país. Una de las características de estos centros es que los usuarios financieros son extranjeros, y las causas pueden ser: las bajas o nulas tasas, moderada regulación financiera y anonimato a las transacciones financieras. 
El capital no reportado da lugar a anomalías en las estadísticas externas. Los autores argumentan que en la mayoría de los casos es una cadena de operaciones que involucra organizaciones como fideicomisos, fundaciones y compañías ficticias que simplemente buscan desvirtuar a inversionistas individuales con altos patrimonios netos. Las formas más convencionales de hacer este tipo de trasferencias de capital que los autores señalan son:

Transferencias de efectivo: implican el cruce de frontera por parte de la persona que posee el dinero, por lo general requieren el traslado a zonas cercanas entre sí, y de esta forma no existe rastro de la transacción.

Facturación de servicios no prestados o bienes inexistentes: este tipo de transacciones se realizan con más frecuencia en zonas donde los impuestos a la renta son muy bajos o donde las restricciones comerciales solamente se enfocan a las actividades internas, de tal forma que no existe control sobre las relaciones comerciales con otras jurisdicciones o países. En esos casos existe poca relación entre el precio, las cantidades y los montos pagados, lo cual también implica una conducta colusoria entre las organizaciones.

Transferencias a entidades extraterritoriales: en algunos casos las organizaciones solicitan a sus clientes realizar sus pagos mediante transferencia de fondos o pagos directos a entidades extraterritoriales.

Los autores señalan que en las dos últimas décadas el crecimiento de las transacciones financieras internaciones ha contribuido a la fuga de capital a centros financieros extraterritoriales en los cuales el secreto bancario y financiero es imperante.

Hanlon, Maydew y Thornock (2015) realizan un estudio en el cual analizan este mismo problema en Estados Unidos: estudian las inversiones y un fenómeno que denominan "ida y vuelta" en el que algunos estadounidenses ocultan fondos en entidades de paraísos fiscales y luego se reinvierten en bonos de EE. UU. Al hacer parecer que las inversiones provienen de inversionistas extranjeros, se evade una parte de los ingresos que genera la inversión en razón a que los inversionistas extranjeros tienen tasas más favorables que los inversionistas locales. 


\subsection{Evasión medida desde la información financiera de las entidades financieras}

Artavanis, Morse y Tsoutsoura (2016) investigan la evasión fiscal tomando como referencia los créditos otorgados a los bancos, con el propósito de determinar los ingresos que implican las capacidades de crédito de la muestra seleccionada. Este estudio se centra en préstamos internos y se estima que los ingresos no declarados en Grecia en 2009 podían ser alrededor de los 26800 millones de ingresos y sobre estos se dejaron de percibir ingresos fiscales del $40 \%$ que es la tasa impositiva en este país; los autores mediante este estudio señalan los profesionales altamente educados y con participaciones gremiales en el parlamento de este país.

Balafoutas, Beck, Kerschbamer y Sutter (2015) desarrollan una investigación en la cual toman como referencia el mercado de los bienes de créditos e identifican que un individuo es más propenso a evadir impuestos cuando existe la posibilidad de colusión por parte de los agentes de un mercado. Esta colusión que se puede dar se identifica como ineficiencias en el mercado, lo cual genera que estos sean asimétricos. Los autores definen este fenómeno como los costos ocultos de la evasión y señalan que este trabajo tiene una contribución significativa en razón a que no solo hace alusión a que la evasión produce una distribución injusta en la carga tributaria, sino que ocasiona costos a la administración tributaria, la cual busca detectar estas actividades en las cuales se evaden los impuestos.

Para Giombini, Teobaldelli y Schneider (2018), el impacto marginal de la evasión fiscal para el apalancamiento financiero de una empresa genera restricciones en la disminución de la eficiencia del sistema legal; es decir, la evasión fiscal y la ineficiencia judicial se mitigan mutuamente; de ahí que esto tiene efecto sobre las restricciones de crédito.

\subsection{Medición de la evasión}

La evasión fiscal es la ocultación ilegal de una actividad sujeta a impuestos. Medir la cantidad de actividad económica que se oculta siempre será difícil, 
ya que quienes se involucran en la evasión tienen todas las motivaciones para ocultar sus actividades. Aun así, las estimaciones disponibles de fuentes oficiales y de investigadores académicos coinciden en que la evasión es una actividad económicamente significativa (Hashimzade et al., 2012).

Diferentes modelos han sido elaborados en la perspectiva de medir los niveles de evasión. Cada uno de estos modelos responde a la perspectiva desde la cual se está intentando medir la evasión, por lo cual, de una manera bastante general, es posible clasificarlos en tres tipos: a) aquellos que toman como punto de referencia el comportamiento de los contribuyentes; b) aquellos desde la perspectiva del comportamiento de las empresas; y, c) aquellos desde el ámbito de la economía como un todo. En lo que sigue se destacan algunos de estos modelos aplicados en estudios recientes.

Los dos primeros grupos de modelos de medición de la evasión tributaria suelen ser identificados como sistemas parciales, micromediciones o enfoques directos referidos a programas específicos de auditoría o fiscalizaciones concretas, las cuales basadas en una muestra considerada como significativa infieren comportamientos para un grupo determinado de contribuyentes. Las metodologías del tercer grupo (c) se basan en mediciones macro o efectos indirectos, a partir de agregados económicos provenientes de las cuentas nacionales o de encuestas de hogares por medio de las cuales se relaciona la recaudación con el uso de determinados insumos físicos utilizados en la producción de bienes y servicios (Gómez y Morán, 2016). En lo que sigue, se destacan algunos de los estudios más recientes vinculados a estos tres tipos de metodologías o modelos de evasión tributaria.

Artavanis (2015) examina la relación del efecto de las tasas impositivas sobre la evasión fiscal, para lo cual estudia el caso del efecto de los cambios en las tasas del impuesto al valor agregado (IVA) sobre la industria griega de restaurantes en un entorno con oportunidades limitadas de transferencia de impuestos. En este estudio se elabora una metodología de cálculo de diferencia en la diferencia (difference-in-difference-DD) basada en el nivel de ventas de empresas pequeñas y grandes tratadas por separado y que permite determinar que una reducción de la tasa de ventas del IVA 
aumenta significativamente el cumplimiento, en especial para las empresas con más oportunidades de evasión.

Barrero et al. (2015) aplican una metodología para estimar el fraude en el Impuesto sobre la Renta de las Personas Físicas (IRPF) en España, que se basa en la detección de comportamientos distintos (por ejemplo, en el consumo) por parte de individuos que obtienen una renta de la misma cuantía, pero procedente de distinta fuente. Esa diferencia de comportamiento informa - según determinadas hipótesis - de la existencia y el importe del fraude cometido.

Bertotti y Modanese (2016) construyen un modelo matemático-cinético, que describe las interacciones económicas, los impuestos y la redistribución en una sociedad cerrada. El modelo tiene en cuenta la presencia de un sistema tributario combinado con un proceso de redistribución, así como la ocurrencia de evasión fiscal dentro del cual se asume la evasión de impuestos en diversos grados admitiendo la posibilidad de que diferentes ciudadanos paguen porcentajes distintos de los impuestos que deberían pagar. El modelo toma en cuenta que la evasión fiscal requiere de una gran cantidad de agentes que interactúan, entre ellos los propios evasores y los ciudadanos. En vista de esto y de los diversos niveles en los que puede tener lugar, la evasión fiscal puede considerarse como un ejemplo de sistema complejo.

Brum y Crokidakis (2016) proponen un modelo de evasión fiscal basado en agentes divididos en tres estados: contribuyentes honestos, evasores de impuestos y aquellos susceptibles de convertirse en evasores. Las transiciones entre las clases se rigen por probabilidades, de manera similar a lo que sucede en los modelos de propagación epidémica. Las transiciones entre estas clases se rigen por cuatro probabilidades distintas, que representan las interacciones sociales y el régimen de aplicación. El modelo elaborado realiza simulaciones en gráficos totalmente conectados, así como en redes complejas aleatorias y sin escala. Para los casos de grafos aleatorios y completamente conectados, se observa que la aparición de evasores de impuestos en la población se asocia con una transición de fase de desequilibrio de absorción activa, que está ausente en las redes libres de escala. 
Gokalp et al. (2017) utilizan un modelo bastante sencillo en el que partiendo de datos de encuestas miden la evasión de impuestos como el porcentaje de ventas no informadas a la autoridad tributaria. Dado que este es un tema delicado, cuestionar directamente a los encuestados no proporcionaría resultados fiables. Por lo tanto, los investigadores formulan las preguntas de manera indirecta y alentaron a más gerentes a participar. Las empresas de la muestra utilizadas para este estudio presentan una gran variación en su comportamiento de evasión tributaria con una tasa promedio de evasión fiscal del 17 \% y una desviación estándar del $26 \%$.

En el caso particular de América Latina, metodologías estándar que permitan una cuantificación de la evasión, así como su explicación al relacionarla con variables de comportamiento es aún una tarea pendiente. Son muy pocos los países de la región (Chile, Colombia, Uruguay, México) que realicen mediciones periódicas que permitan cuantificar el nivel de incumplimiento tributario a través del tiempo, aunque estas mediciones están referidas principalmente al IVA e impuesto a la renta, y quedan al margen el resto de tributos.

En cuanto al IVA, tal y como destacan Gómez y Morán (2016), los estudios realizados por las propias administraciones tributarias o investigadores independientes, uno de los métodos más utilizados es el del potencial tributario, el cual determina un valor de recaudación potencial del tributo comparándolo luego con el valor efectivamente recaudado, y la brecha resultante entre ambos es el valor estimado de evasión fiscal. En lo que respecta al impuesto a la renta empresarial, la metodología usualmente utilizada en los estudios regionales estima la recaudación teórica del impuesto a partir del excedente de explotación de cuentas nacionales. Por otro lado, para el cálculo de la evasión en el impuesto a la renta de las personas físicas, las metodologías más utilizadas son aquellas basadas en la estimación de la recaudación potencial a partir de los datos de las encuestas de hogares.

Como se desprende del trabajo de Jorrat y Podestá (2010), el cual realiza un recuento de las diferentes metodologías utilizadas para el cálculo de la evasión de impuestos, en la mayoría de los casos los resultados son muy 
sensibles a la metodología aplicada, además que la falta de un consenso impide la comparabilidad entre países de los valores estimados, y es la elaboración de una técnica estándar uno de los principales desafíos para la región en materia tributaria. En este sentido, como un referente importante, la Comisión Europea viene publicando una serie de estudios —el más reciente corresponde a 2017- tendientes a cuantificar la evasión tributaria en el IVA para los países de la región y facilitar la comparación entre ellos (Gómez y Morán, 2016).

\section{Conclusiones}

Los ejercicios de revisión bibliográfica son usualmente provechosos para académicos y estudiosos del tema; sin embargo, es importante resaltar que frente a la evasión tributaria se tornan valiosos no solo para la academia sino también para otros actores como el Estado, los entes de control y se esperaría que los ciudadanos, justamente por ser este un tema transversal a la gestión administrativa de los recursos públicos y por ende a la política fiscal, en relación con sus impactos en la distribución del ingreso y en la eficiencia en el recaudo, a través de la figura de los impuestos, sean estos directos como el impuesto de renta, o indirectos como el IVA.

Con ese propósito, la evasión es entendida como el incumplimiento de las obligaciones tributarias, producto de diversos aspectos que van desde la inconformidad de los ciudadanos hasta la falta de legitimidad del Estado, impactando poderosamente las dinámicas sociales de las diferentes economías mundiales en las cuales se desarrolla el estudio, así como en otros contextos. A partir de lo anterior, se evidencia un sinnúmero de estrategias que buscan mitigar las causas que generan la evasión tributaria, apuntando a cruces de información entre países, al igual que a la utilización de métodos, tanto directos como indirectos, que permitan cuantificar este fenómeno y de esa forma propiciar dinámicas de control.

No obstante, y a partir de la categorización posterior a la recolección y sistematización de los artículos académicos sobre la evasión tributaria, se 
encuentra principalmente que sobre el tema los avances giran en torno a: estudios relacionados con las causas de los comportamientos evasores y caracterización de los factores positivos y negativos que inciden en la conducta de los evasores. Por otro lado, se encuentran investigaciones que analizan las justificaciones que esbozan los contribuyentes para evadir sus obligaciones tributarias clasificándolas en niveles internos, contextuales y sociales. En cuanto a los beneficios de los evasores, diferentes investigaciones indican que la evasión de impuestos contribuye a incrementar las utilidades de las empresas evasoras, propiciando la entrada o salida de empresas a un mercado. Al margen, se encuentran los estudios relacionados con los paraísos fiscales como espacios de evasión internacional, contribuyendo a la fuga de capital. Con respecto al control de la evasión, la auditoría tributaria se torna como uno de los medios más efectivos para el control fiscal, de la mano del uso tecnológico, los cruces de información y la eficiencia en la redistribución de la riqueza, entre otros.

Entre los diferentes resultados se pueden destacar como una modificación incremental en las tasas, lo cual implica un aumento en el nivel de evasión, mientras que la disminución de la evasión, mediante los cruces de información, garantiza la reducción en los recursos que se destinaban para su control. Puede afirmarse que los actuares injustos socialmente, como altas cargas tributarias a pequeños contribuyentes, ocasionan fuertes desigualdades que debilitan el sistema tributario y motivan a los contribuyentes a no cumplir con sus obligaciones. Por último, la importancia de la cultura ciudadana frente al sistema tributario demuestra que cuando el bienestar y la percepción que se tiene de las instituciones y de los agentes del Gobierno (policía, democracia, gestión pública, etc.) es positiva, la evasión tiende a reducirse.

Finalmente, la evidencia documental comprueba que los sistemas tributarios de los contextos analizados implican la definición de políticas fiscales particulares que terminan determinando los comportamientos de los diferentes contribuyentes, al igual que el de los Estados y el de las instituciones gubernamentales relacionadas con el control del recaudo de los impuestos. 


\section{Referencias}

Abdixhiku, L., Pugh, G. y Hashi, I. (2018). Business tax evasion in transition economies: a cross-country panel investigation. The European Journal of Comparative Economics, 15(1), junio, 11-36. Cattaneo University (LIUC).

Alarcón, G., Buendía, J. y Sánchez, M. (2017). Tax evasion in europe: an analysis based on spatial dependence. Social Science Quarterly, 99(1), 1-17.DOI: https:// doi.org/10.1111/ssqu.12382

Argentiero, A., Chiarini, B. y Marzano, E. (2015). Tax evasion and economic crime. empirical evidence for Italy. CESIFO Working Paper Series, (5497), 1-36. CESifo Group Munich

Artavanis, N., Morse, A. y Tsoutsoura, M. (2016). Measuring income tax evasion using bank credit: evidence from Greece. The Quarterly Journal of Economics, 131(2), mayo, 739-798. DOI: https://doi.org/10.1093/qje/qjw009

Balafoutas, L., Beck, A., Kerschbamer, R. y Sutter, M. (2015). The hidden costs of tax evasion. Collaborative tax evasion in markets for expert services. Journal of Public Economics, 129, septiembre, 14-25. DOI: https://doi.org/10.1016/j. jpubeco.2015.06.003

Banerjee, D. y Vaidya, S. (2018). Anticorruption reforms, tax evasion, and the role of harassment. Journal of Public Economic Theory, (21), 62-80. DOI: https:// doi.org/10.1111/jpet.12304

Bernasconi, M., Levaggi, R. y Menoncin, F. (2016). Dynamic tax evasion with habit formation. Department of Economics Working Paper, (31), 1-24. Recuperado de https://www.unive.it/pag/fileadmin/user_upload/dipartimenti/economia/ doc/Pubblicazioni_scientifiche/working_papers/2016/WP_DSE_bernasconi_levaggi_menoncin_31_16.pdf

Bertotti, H. y Modanese, G. (2016). Mathematical models describing the effects of different tax evasion behaviors. Journal of Economic Interaction and Coordination, 13(2), julio, 351-363. Springer: Society for Economic Science with Heterogeneous Interacting Agents. DOI: https://doi.org/10.1007/ s11403-016-0185-9

Bobbio, E. (2016). Tax evasion, firm dynamics and growth. Questioni di Economia e Finanza (Occasional Papers), 357. Bank of Italy, Economic Research and International Relations Area. 
Brum, R. y Crokidakis, N. (2016). Dynamics of tax evasion through an epidemic-like model. International Journal of Modern Physics C, 28(2), 1-14. DOI: https:// doi.org/10.1142/S0129183117500231

Casaburi, L. y Toriano, U. (2015). Ghost-house busters: the electoral response to a large anti tax evasion program. Nber Working Paper 21185. Cambridge: NBER. Recuperado de https://www.nber.org/papers/w21185.pdf

Cosulich, J. (1993). La evasión tributaria. Santiago de Chile: Cepal. Recuperado de https://repositorio.cepal.org/bitstream/handle/11362/9480/ S9300143_es.pdf?sequence $=1$ \&isAllowed $=y$

Deslauriers, J. P. (2004). Investigación cualitativa: guía práctica. Pereira: Universidad Tecnológica de Pereira. Recuperado de http://repositorio.utp.edu.co/dspace/ bitstream/handle/11059/3365/Investigaci\%c3\%b3n\%20cualitativa.\%20pdf. $\mathrm{PDF}$ ? sequence $=4$ \&isAllowed $=\mathrm{y}$

Dularif, M., Nurkholis Sutrisno, T. y Saraswati, E. (2019). Is deterrence approach effective in combating tax evasion? A meta-analysis. Problems and Perspectives in Management, 17(2), abril, 93-113. DOI: https://doi.org/10.21511/ ppm.17(2).2019.07

Ferrer, A. y Gërxhani, K. (2016). Tax evasion and well-being: a study of the social and institucional context in Central and Eastern Europe. European Journal of Political Economy, 45, Supplement, diciembre, 149-159. DOI: https://doi. org/10.1016/j.ejpoleco.2016.09.004

Fochmann, M. y Wolf, N. (2019). Framing and salience effects in tax evasion decisions: an experiment on underreporting and overdeducting. Journal of Economic Psychology, 72, junio, 260-277. DOI: https://doi.org/10.1016/j. joep.2019.03.005

Giombini, G., Teobaldelli, D. y Schneider, F. (2018). Interaction effect of tax evasion and legal system inefficiency on firms' financial constraints. International Review of Economics and Finance, 55(C), 1-20. DOI: 10.1016/j.iref.2018.01.009

Goerke, L., Bonn, I. y München, C. (2016). Tax evasion in a cournot oligopoly with endogenous entry. International Tax and Public Finance, 24(5), 754-779. DOI: https://doi.org/10.1007/s10797-016-9434-Z

Gokalp, O. N., Leeb, S.-H. y Pengb, M. W. (2017). Competition and corporate tax evasion: an institution-based view. Journal of World Business, 52(2), enero, 258-269. DOI: https://doi.org/10.1016/j.jwb.2016.12.006 
Gómez, J. y Morán, D. (2016). Evasión tributaria en América Latina: nuevos y antiguos desafíos en la cuantificación del fenómeno en los países de la región. Santiago de Chile: Cepal. Recuperado de https://repositorio.cepal.org/ bitstream/handle/11362/39902/1/S1600017_es.pdf

Goumagias, N., Hristu-Varsakelis, D. y Assael, Y. (2018). Using deep Q-learning to understand the tax evasion behavior of risk-averse firms. Expert Systems With Applications: An International Journal, 101(C), julio, 258-270. Elmsford, Estados Unidos: Pergamon Press. DOI: https://doi.org/10.1016/j. eswa.2018.01.039

Hanlon, M., Maydew, E. L. y Thornock, J. R. (2015). Taking the long way home: U.S. tax evasion and offshore investments in U.S. equity and debt markets. The Journal of Finance, 70(1), enero, 257-287. Estados Unidos: American Finance Association/Wiley. Recuperado de https://dspace.mit.edu/bitstream/ handle/1721.1/98848/Hanlon_Taking\%20the.pdf?sequence $=1$ \&isAllowed $=y$

Hashimzade, N., Gareth, M. y Tran-Nam, B. (2012). Applications of behavioural economics to tax evasion. Journal of Economics Surveys, 27(5), 941-977. DOI: https://doi.org/10.1111/j.1467-6419.2012.00733.x

Henry, E., Massel, N. y Towery, E. (2016). Increased tax disclosures and corporate tax avoidance. National Tax Journal, 69(4), diciembre, 809-830. DOI: https:// doi.org//10.17310/ntj.2016.4.04

Hernández, R., Collado, C. y Baptista, P. (2010). Metodología de la investigación (quinta edición). México: McGraw-Hill. Recuperado de: https://www. esup.edu.pe/descargas/dep_investigacion/Metodologia\%20de $\% 201 \mathrm{la} \% 20$ investigaci\%C3\%B3n\%205ta\%20Edici\%C3\%B3n.pdf

Hoopes, J. L., Thornock, J. R. y Williams, B. M. (2016). Does use tax evasion provide a competitive advantage to e-tailers? National Tax Journal, 69(1), 133-168. DOI: https://doi.org//10.17310/ntj.2016.1.05

Immordino, G. y Flaviano Russo, F. (2018). Cashless payments and tax evasion. European Journal of Political Economy, 55(C), 36-43. DOI: https://doi. org/10.1016/j.ejpoleco.2017.11.001

Ionel, L. (2016). The effectiveness of control on combating tax evasion and fraud at national level. Social Economic Debates, 5(2), 16-22.

Jiménez, J., Gómez, J. y Podestá, A. (comps.). (2010). Evasión y equidad en América Latina. Santiago de Chile: Cepal. Recuperado de https://www.cepal.org/ilpes/ noticias/paginas/5/38375/Evasi\%C3\%B3n_y_equidad_final_02-02-2010.pdf 
Jorrat, M. y Podestá, A. (2010). Análisis comparativo de las metodologías empleadas para la estimación de la evasión en el impuesto de renta. En J. Jiménez, J. Gómez y A. Podestá, Evasión y equidad en América Latina (pp. 69-94). Santiago de Chile: Cepal. Recuperado de https://www.cepal.org/ilpes/noticias/ paginas/5/38375/Evasi\%C3\%B3n_y_equidad_final_02-02-2010.pdf

Kuchumova, Y. (2017). The optimal deterrence of tax evasion: the trade-off between information reporting and audits. Journal of Public Economics, 145, enero, 162-180. DOI: https://doi.org/10.1016/j.jpubeco.2016.11.007

López, J. y Sanz, E. (2016). La moral fiscal de los españoles, reexaminada. Revista de Economía Aplicada, 24(70), 53-76.

Maji, S. (2017). Can tax evasion be justified?: a behavioural study. DOI: http:// dx.doi.org/10.2139/ssrn.3024959

McGee, R., Devos, K. y Benk, S. (2016). Attitudes towards tax evasion in Turkey and Australia: a comparative study. Social Sciences, 5(1), marzo, 1-13.

McGee, R., Yoon, Y. y Li, Y. (2015). The ethics of tax evasion a comparative study of Chinese and U.S. opinions. Indonesian Journal of International \& Comparative Law, 2(1), 161-186.

Mityakov, S. (2015). Offshore schemes and tax evasion: the role of banks. DOI: https://doi.org/10.13140/RG.2.1.3916.2727

Nygard, O. E., Slemrod, J. y Thoresen, T. O. (2016). Distributional implications of joint tax evasion. CESifo Working Paper Series 5915. CESifo Group Munich.

OECD. (2015). In it together: why less inequality benefits all. Overview of inequality trends, key findings and policy directions. París: OECD Publishing. Recuperado de https://www.oecd.org/els/soc/OECD2015-In-It-Together-Chapter1Overview-Inequality.pdf

Parra, O. y Patiño, R. (2010). Evasión de impuestos nacionales en Colombia: años 2001-2009. Revista Facultad de Ciencias Económicas: Investigación y Reflexión, XVIII(2), diciembre, 177-198. Recuperado de http://www.scielo.org.co/pdf/ rfce/v18n2/v18n2a11.pdf

Pellegrini, V., Sanelli, A. y Tosti, E. (2016). What do external statistics tell us about undeclared assets held abroad and tax evasion? Bank of Italy Occasional Paper, (367). DOI: http://dx.doi.org/10.2139/ssrn.2917184

Piolatto, A. (2014). Itemised deductions: a device to reduce tax evasion. German Economic Review, 16(4), 422-438. DOI: https://doi.org/10.1111/geer.12059 
Porter, T. y Ronit, K. (2018). Whistleblowing as a new regulatory instrument in global governance: the case of tax evasion. Cambridge Review of International Affairs, 31(6), 537-560. DOI: https://doi.org/10.1080/09557571.2018.1546278

Saxunova, D. y Szarkova, R. (2018). Global efforts of tax authorities and tax evasion challenge. Journal of Eastern Europe Research in Business and Economics, 2018, 1-14. Article ID 511388. DOI: https://doi.org/10.5171/2018.511388

Shome, P. y Tanzi, V. (1993). Tax evasion: causes, estimation methods, and penalties. A focus on Latin America. Santiago de Chile: Cepal. Recuperado de http://repositorio.cepal.org/bitstream/handle/11362/9464/ S9300142_en.pdf?sequence=1\&isAllowed=y

Sidani, Y., Ghanem, A. y Rawwas, M. (2014). When idealists evade taxes: the influence of personal moral philosophy on attitudes to tax evasion - a Lebanese study. Business Ethics: A European Review, 23(2), 183-196. DOI: https://doi. org/10.1111/beer.12046

Strauss, A. y Corbin, J. (2002). Bases de la investigación cualitativa. Técnicas y procedimientos para desarrollar una teoría fundamentada. Medellín: Universidad de Antioquia. Recuperado de https://diversidadlocal.files.wordpress. com/2012/09/bases-investigacion-cualitativa.pdf

Subdirección General de Asuntos Económicos y Financieros de la Unión Europea. (2013). Avances de la Unión Europea en la lucha contra el fraude y la evasión fiscal. El intercambio automático de información. Boletin Económico de ICE (Información Comercial Española), (3046), diciembre, 3-18.

Watson, L. (2015). Corporate social responsibility, tax avoidance, and earnings performance. The Journal of the American Taxation Association, 37(2), 1-21. DOI: https://doi.org/10.2308/atax-51022

Zídková, H., Tepperová, J. y Helman, K. (2018). EVS data-based analysis of tax evasion: descriptive vs. regression modelling. Society and Economy, 40(1), marzo, 89-103. 